\title{
Traduire
}

Ine autre perspective sur I t raduction

Revue française de la traduction

$232 \mid 2015$

Intraduisible? Vous voulez rire !

\section{Seleskovitch et M. Lederer : Interpréter pour traduire}

Freddie Plassard

\section{(2) OpenEdition}

1 Journals

Édition électronique

URL : http://journals.openedition.org/traduire/717

DOI : $10.4000 /$ traduire. 717

ISSN : 2272-9992

Éditeur

Société française des traducteurs

Édition imprimée

Date de publication : 15 juin 2015

Pagination : 141-143

ISSN : 0395-773X

\section{Référence électronique}

Freddie Plassard, « D. Seleskovitch et M. Lederer : Interpréter pour traduire », Traduire [En ligne], 232 |

2015, mis en ligne le 15 juin 2015, consulté le 23 septembre 2020. URL : http://

journals.openedition.org/traduire/717 ; DOI : https://doi.org/10.4000/traduire.717 


\section{Compte rendu d'ouvrage D. Seleskovitch et M. Lederer : Interpréter pour traduire}

\section{Freddie Plassard}

La cinquième édition d'Interpréter pour traduire, recueil d'articles initialement publiés dans différentes revues et rassemblés en un volume paru pour la première fois en 1984, réédité en 2001 sous les auspices de l'Union européenne, vient de paraître dans la collection de Traductologie que dirige J.-R. Ladmiral aux éditions des Belles Lettres. Pour présenter cette édition, M. Lederer a tenu une conférence dans les locaux de I'ISIT à l'intention d'un public d'enseignants et d'étudiants. Ce fut pour elle l'occasion de jeter un regard à la fois rétrospectif et prospectif sur un ouvrage central de la traductologie de langue française.

Rappelant la structure de l'ouvrage en trois chapitres : Qu'est-ce que traduire ? L'enseignement de l'interprétation et La traduction et le langage, elle n'a pas manqué de souligner ce que la trentaine d'années écoulées depuis la première publication nous a sans doute fait oublier, tel le caractère précocement interdisciplinaire de la recherche sur l'interprétation, où neuropsychologie et psychologie génétique sont venues étayer les constats et observations faits en situation. Rapidement convaincues par la pratique de l'interprétation de conférences, que la linguistique, celle de Saussure, Martinet, Jakobson ou encore Mounin, n'était pas la voie à suivre pour décrire et expliquer la traduction, M. Lederer et D. Seleskovitch formulent quelques constats fondamentaux : la traduction est une opération de communication et non sur les signes linguistiques, assimilable en première approche à toute opération de communication unilingue. Si cette inscription de la pratique dans le cadre plus large de la communication ne nous étonne plus, les travaux sur l'interaction et les échanges conversationnels ne se sont développés, eux, qu'à compter des années 1980 aux États-Unis et plus tard en France, souligne M. Lederer. Ce recadrage est allé de pair avec une prise de position théorique dont les effets ne sont, selon elle, pas toujours pris en compte en traductologie : la différence entre langue d'une part et discours de l'autre, discours lui-même situé, contextualisé, jugulant par là-même la multiplication de virtualités de sens. Tout aussi capitale est la description du processus de traduction en trois temps. Outre la compréhension et la reformulation, les deux auteurs ont en effet dégagé une phase de déverbalisation où disparaît l'" enveloppe linguistique initiale " de l'énoncé, battant en brèche toute approche contrastive de la traduction qui reposerait sur la comparaison des langues dites en contact. 
Pionnières, D. Seleskovitch et M. Lederer ont pris position sur des sujets en des termes qui se verront les uns après les autres confortés par des figures d'autorité de la sphère intellectuelle :

- Le fait que la compréhension repose par principe sur l'adjonction à tout énoncé de connaissances extra-linguistiques sans lesquelles ces énoncés resteraient abscons, autrement dit l'impossibilité de s'en tenir au seul donné linguistique pour comprendre et le rôle capital de la coopération du récepteur qui active et mobilise ses propres connaissances, fait corroboré par nombre d'études ultérieures (U. Eco(1), P. Grice(2), D. Sperber et E. Wilson(3)).

- Le fait que la compréhension du langage amène très vite à ne saisir qu'un sens à la fois, fait conforté par les travaux de S. Dehaene(4), mais aussi par les études cognitives de la compréhension à la lecture(5).

- L'usage réflexe du langage dès lors que les locuteurs s'expriment dans leur propre langue ou dans une langue réellement maîtrisée, avec pour corollaire la possibilité de dissocier pensée consciente et usage du langage en grande part réflexe, forme d'autonomie des processus mentaux que confortent la visualisation des zones d'activation du cerveau par imagerie cérébrale et les travaux de S. Pinker(6) et D. Laplane(7).

- Le fait que la compréhension s'établit selon un processus descendant, partant de la globalité du texte pour aller vers ses constituants et non l'inverse.

- Le fait que dans toute langue, toute réalité est exprimée par des dénominations qui procèdent par sélection de traits de signification, principe de synecdoque, avec pour contrepartie, en discours, le fait que seule une partie de ce qui est visé par un énoncé s'y trouve explicitée, laissant dans l'ombre une part implicite qui n'en contribue pas moins à l'intelligibilité du sens.

(1) Eco, Umberto (1993) : Lector in fabula, Le rôle du lecteur ou la coopération interprétative dans les textes narratifs, Paris, Grasset, Le livre de poche. Titre original : Lector in fabula, Milan, Bompiani, 1979, traduit de l'italien par M. Bouzaher 1re édition de la traduction, 1985, Paris, Grasset \& Fasquelle.

(2) Grice, H. Paul (1967) : 'Logic and Conversation', tapuscript des William James Lectures, Harvard University. Publié dans Grice (1989), Studies in the Way of Words, Harvard University Press, Cambridge, Massachusetts, p. 22-40.

(3) Sperber, Dan et Wilson, Deirdre (1986) : Relevance - Communication and Cognition, Oxford, Basil Blackwell.

(4) S. Dehaene, Stanislas (2014) : Le code de la conscience, Paris, Odile Jacob.

(5) Voir en particulier : Plassard, Freddie (2005) : "Sens ou représentation ? Étude comparative de la compréhension selon les modèles cognitifs de la lecture et le modèle interprétatif de la traduction ", in Israël F. et Lederer M. (dir.), La théorie interprétative de la traduction : regards croisés, vol. II, Paris, Minard, p. 99-133 ; (2007) : Lire pour traduire, Paris, Presses Sorbonne Nouvelle.

(6) Pinker Steven (1994) : The Language Instinct - The New Science of Language and Mind, Londres, Penguin Books.

(7) Laplane Dominique (1997) : La pensée d'outre-mots - la pensée sans langage et la relation pensée-langage, Institut Sanofi-Synthélabo, Paris, 1997 et (2005) Penser, c'est-à-dire ? Enquête neurophilosophique, Paris, Armand Colin. 
À cette liste d'arguments plaidant en faveur du caractère novateur de la théorie interprétative au moment de sa formulation, M. Lederer ajoute un nouvel argument, celui de l'importance du raisonnement analogique pour la compréhension. Là où la tentative de repérage d'analogies ou de différences entre les langues n'apporte rien à la connaissance de la traduction, le raisonnement analogique joue, lui, un rôle important dans la compréhension en général et celle de sujets spécialisés en particulier, le fait de rapporter un élément nouveau à quelque chose de connu permettant de l'" apprivoiser".

Si l'auditeur a été convaincu par l'exposé oral, le lecteur le sera-t-il autant à la lecture de lignes lui rappelant les dérives méthodologiques du comparatisme " induit par le caractère fixé du texte écrit " (p. 4), sachant d'une part la véritable explosion des études empiriques consacrées à la réalisation écrite du processus de traduction(8) et, d'autre part, le caractère désormais ô combien provisoire des textes transmis aux traducteurs qui ne manquent pas de le déplorer(9) ?

D. Seleskovitch et M. Lederer, Interpréter pour traduire, 5e édition revue et corrigée, 2014, Les Belles Lettres, Préface de J.-R. Ladmiral, (1re édition, 1984).

(8) Voir notamment Foz, Clara et Fraser, Ryan (dir.) (2011) : Cartographie des méthodologies de traduction, TTR, vol. XXIV n².

(9) Voir C. Durban : Faire les bons choix, Petit guide de l'acheteur de traductions, Paris, SFT, rubrique "Finalisez votre texte avant de démarrer la traduction ". 